\title{
Aberrant DNA Methylation Characterizes a Subtype of Juvenile Myelomonocytic Leukemia (JMML) with Poor Outcome.
}

Christiane Batz, MS ${ }^{*}, 1$, Inga Sandrock, BS ${ }^{*}, 1$, Peter Nöllke, $\mathrm{MPH}^{*}{ }^{* 1}$, Brigitte Strahm, MD ${ }^{*, 1}$, Henrik Hasle, $\mathrm{MD}^{2}$, Marco Zecca, MD ${ }^{*, 3}$, Jan Starý, MD ${ }^{*, 4}$, Eva Bergsträsser, MD ${ }^{*, 5}$, Barbara De Moerloose, $\mathrm{MD}^{*, 6}$, Monika Trebo, MD, PhD ${ }^{*, 7}$, Marry M. van den Heuvel, MD, PhD ${ }^{*, 8}$, Dorota Wojcik, MD ${ }^{*, 9}$, Franco Locatelli, MD ${ }^{*}, 10$, Charlotte M Niemeyer, MD $^{1}$ and Christian Flotho, $\mathrm{MD}^{*}, 1$

${ }^{1}$ Dept. of Pediatrics and Adolescent Medicine, University Medical Center, Freiburg, Germany,

${ }^{2}$ Dept. of Pediatrics, Aarhus University Hospital Skejby, Aarhus, Denmark,

${ }^{3}$ Pediatric Hematology-Oncology, Fondazione IRCCS Policlinico San Matteo, Pavia, Italy,

${ }^{4}$ CLIP Dept. of Pediatric Hematology and Oncology, Charles University Prague, Prague, Czech Republic,

${ }^{5}$ Dept. of Pediatric Hematology-Oncology, University Children's Hospital, Zürich, Switzerland,

${ }^{6}$ Dept. of Pediatric Hematology-Oncology, University Hospital, Ghent, Belgium,

${ }^{7}$ Dept. of Pediatrics, St. Anna Children's Hospital, Vienna, Austria,

${ }^{8}$ Dept. of Pediatric Oncology-Hematology, Erasmus Medical Center, Rotterdam, Netherlands,

${ }^{9}$ Dept. of Pediatric Hematology-Oncology, Wroclaw Medical University, Wroclaw, Poland,

${ }^{10}$ Pediatric Hematology-Oncology, University of Pavia Fondazione IRCCS Policlinico San Matteo, Pavia, Italy

\section{Abstract 828}

Promoter DNA hypermethylation contributes to the malignant phenotype in cancer including myeloproliferative neoplasms and myeloid leukemia. We hypothesized that aberrant DNA methylation also occurs in juvenile myelomonocytic leukemia (JMML) and asked whether it is associated with clinical, hematologic or prognostic features of the disease. Denaturing liquid chromatography was used to analyze peripheral blood or bone marrow samples from 87 children with JMML and 17 healthy control subjects for changes in DNA methylation at 14 candidate gene loci (BMP4, CALCA, CDKN1C,CDKN2B, DAPK1, MGMT, MLH1, PAWR, $R A R B, R A S A 1, R A S S F 1, R E C K, S O C S 1, T P 73)$. We identified 4 genes with promoter DNA hypermethylation in JMML: BMP4 (34\% of cases), CALCA (30\%), CDKN2B (28\%) and $R A R B(23 \%)$; the other 10 loci were unmethylated. The pattern of hypermethylation of the 4 genes allowed the categorization of JMML cases into three groups: no methylation (40/87 patients), intermediate methylation ( 1 or 2 genes; 29/87 patients) or high methylation ( 3 or 4 genes; $18 / 87$ patients). Lineage-specific cell sorting demonstrated that aberrant methylation was restricted to clonal cell populations and could be traced back to the CD34+ JMML progenitor cell compartment. This observation supports the concept that DNA methylation is associated with early pathogenetic events in JMML.

A correlative analysis of methylation groups with clinical or hematologic features showed that high methylation was strongly associated with higher age and increased hemoglobin F level at diagnosis (both $\mathrm{p}<0.01$ ). By contrast, there was no significant association with leukocyte count, absolute monocytes, platelet count, blast percentage in blood or bone marrow, spleen size, karyotype or mutational category (PTPN11, RAS, CBL, NF1). Importantly, the presence of hypermethylation at diagnosis predicted cases with poor outcome; the 5-year overall survival (OS) in the no / intermediate / high methylation groups was 0.63 [0.47-0.79] / 0.52 [0.30-0.74] / 0.24 [0.04-0.44] $(\mathrm{p}<0.01)$. Among patients receiving hematopoietic stem cell transplantation, hypermethylation characterized cases with significantly increased probability 
of relapse: the 5-year relapse incidence in the no / intermediate / high methylation groups was $0.22[0.11-0.45] / 0.21[0.09-0.50] / 0.69$ [0.49-0.96] $(\mathrm{p}<0.01)$. The predictive power of high methylation was also reflected in a multivariate Cox model for OS that included age at diagnosis, sex, platelet count and mutational category as other variables; here methylation was the only significant prognostic factor. Furthermore, longitudinal analyses indicated that some cases had acquired a higher methylation phenotype at relapse. In summary, we report aberrant DNA methylation as the most important molecular predictor of outcome in JMML. We suggest that a high-methylation phenotype characterizes an aggressive biologic variant of this leukemia.

Disclosures: No relevant conflicts of interest to declare. 